\title{
Design and development of Taeneb City Guide - From Paper Maps and Guidebooks to Electronic Guides
}

\author{
Dr Mark D Dunlop ${ }^{\mathrm{a}}$, Dr Piotr Ptasinski ${ }^{\mathrm{a}}$, Dr Alison Morrison ${ }^{\mathrm{b}}$, \\ Stephen McCallum ${ }^{\mathrm{a}}$, Chris Risbey ${ }^{\mathrm{b}}$, Fraser Stewart ${ }^{\mathrm{a}}$ \\ ${ }^{a}$ Computer and Information Sciences Department \\ University of Strathclyde, UK \\ mark.dunlop@cis.strath.ac.uk \\ ${ }^{\mathrm{b}}$ Scottish Hotel School \\ University of Strathclyde, UK \\ alison.j.morrison@strath.ac.uk
}

\begin{abstract}
This paper reports the design, development and feedback from the initial trial of the Taeneb City Guide project developing tourist information software on Personal Digital Assistant (PDA) handheld computers. Based on the users' requirements for electronic tourists guides already published in the literature, the paper focuses on the three main technology features of the systems, which would give the advantage over the existing paper publication:

- $\quad$ Query-able dynamic map interface;

- $\quad$ Dynamic information content;

- Community review systems and users' forum.
\end{abstract}

The paper also reports the results of an initial trial of a City Guide for Glasgow conducted as part of the EMAC 03 conference.

Keywords: electronic city guide; PDA applications; handheld usability.

\section{Introduction}

Previous research on tourist behaviour and practices suggest that to be successful, electronic guidebooks must offer compelling advantages over their paper equivalents (e.g. Brown and Chalmers, 2003). Some guidebooks and maps have been already computerised, but with very little penetration into the market place (even for users already owning a palmtop/PDA). Brown and Chalmers suggested that this limited success could be put to a lack of knowledge about how tourists arrange their activities in particular how maps and guidebooks are actually used by tourists. While new 
mobile technologies (with the exception of mobile phones) have been slow to penetrate markets, there are strong niche markets for handheld devices and in the near future combined PDA / "smart" phones are very likely to be in widespread use thus permitting easier deployment of electronic tourist guides on mass market devices. We believe that, given mass market devices, the most important elements of successful tourist information systems are the availability of quality data content and a user interface allowing easy, accurate and quick access to that content. User interface design is a significant challenge for any handheld software development due to the limited power and interaction possibilities (e.g. small screen size and more difficult ways of data entry when compared to desktop interface design) (Brewster and Dunlop, 2000). In our design of Taeneb City Guide we focused on creating an intuitive interface based on requirements and recommendations from previous ethnographic studies on tourist practices and ways of using information sources (Brown and Chalmers, 2003; Brown, and Perry, 2002).

\section{Dynamic Map Display}

Tourists most often use two commonly available types of publications: guidebooks and maps and often use these in combination (Brown and Chalmers, 2003). Guidebooks provide in a standardised and catalogue way information about attractions and places of interest in a city (accommodation, pub/bars, night clubs, restaurants, museums etc.). Maps are used for both localising where things are while searching for particular attractions or browsing the names of attractions for given locations. Using maps with guidebooks solves the problem of where attractions are and what attractions are in a given location. Using guidebooks as a reference, tourists find out on a map where the listed places are located, the approximate distance between two different places and how to navigate from one place to another. Using map as a reference, tourists can search in a guidebook relevant descriptions of places that they found on the map.

The user interface for Taeneb City Guide was designed with two types of views providing both map and list-index

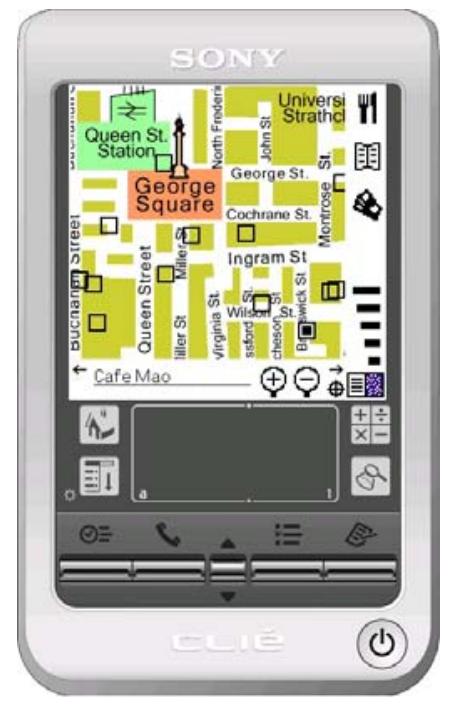

Fig. 1. Map View Display (MVD) 
information access: Map View Display (MVD) and List View Display (LVD).

The main part of the MVD shows a map of a city centre with an overlay set of attractions represented by squares. (see Fig 1.). Users can browse a selected set of attractions by pointing and tapping on symbols of attractions in a selected area. The map interface offers typical electronic map features such as zooming and panning. A user tapping on the display in Fig. 1 over, say, Central Station would zoom the map into that location, a further tap zooms further into that location and then the user can tap on square attraction icons to see the name and then again for more details on that attraction. Users can pan the display by dragging with their stylus. Around the edge of the map in the MPV view are control icons - the most important being a set of filters, here showing type of attractions as restaurant ( $\mathbf{\Psi} \mid \mathbf{1})$, the restaurant type filter (䣵) and the restaurant price filter ( These filters are discussed in more detail later.

LVD is a list displaying the names of attractions in a sorted order in a similar manner to an index at the end of a guidebook (see Fig. 2). An electronic method of presentation can give the advantage over paper editions by providing different sorting criteria (e.g. alphabetic, distance from a given location, relevance according to a search criteria or proximity to previous browsing behaviour).

Simply switching between the displays allows making a quick connection between where attractions are, brief descriptions and reviews.

One of main concerns with using maps on handheld devices is a limited screen size with low density (a typical size of Palm display is $6 \times 6 \mathrm{~cm}$ with a standard resolution of 160x160 - some PalmOS devices now have a 320x320 "high resolution" screens, as used in our study). In order to achieve the best visual performance for our system, we decided to use simplified bitmap/raster formatted maps. The maps were specially drawn for easy reading and use by a tourist while walking. However, it gives the disadvantage over more traditional vector maps of increased storage cost and reduced flexibility in scalability and zooming. While new screens will increase their dots-per-inch resolution, the screen size will remain small by nature thus although high quality digital vectors maps may be possible in the future the design constraints of readability may still require hand-drawn bitmap graphics.

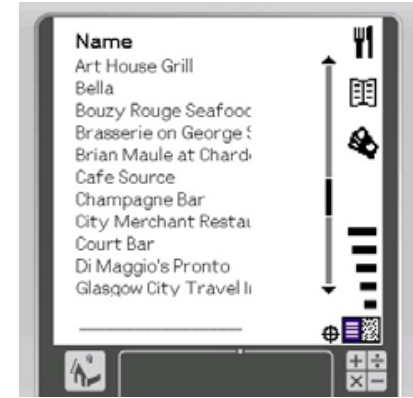

Fig. 2. List View Display (LVD) of Taeneb City Guide 
Brown and Chalmers note that during the studies of tourist behaviour users bookmarked pages in guidebooks by placing fingers into the pages. The Taeneb City guide attempts to support similar style of bookmarking by allowing users to record an attraction as "My Attraction" (and later to see only those attractions). Showing all selected attractions on a MVD can help in easy route planning for a sightseeing walk in a city and, when combined with filters, allows users to see higher level clustering of attractions (discussed below). The system could easily be extended to support automatic route planning functions between attractions, however Brown and Chalmers emphasise that tourists quite often wander randomly, only checking on a map their correct walk direction in order to have a more relaxed visit to a city.

As mentioned above a core functionality of Taeneb City Guide user interface is incorporating dynamic query filters for searching and finding tourist attractions. Query filters are predefined for different types of attractions and designed for rapid selection as pop-up lists for single choice or a separate view with a checklist for multi choice selections (see Fig. 3). For example for restaurants there is a multi-choice filter with a food type (Fig. 3A) and a single choice filter with a price range (Fig. 3B). The results of a query are displayed as a subset of data either as a list using LVD or as a scattered plot of matching attraction-icons on a map display using MVD. The second type of information presentation is known as a starfield display.
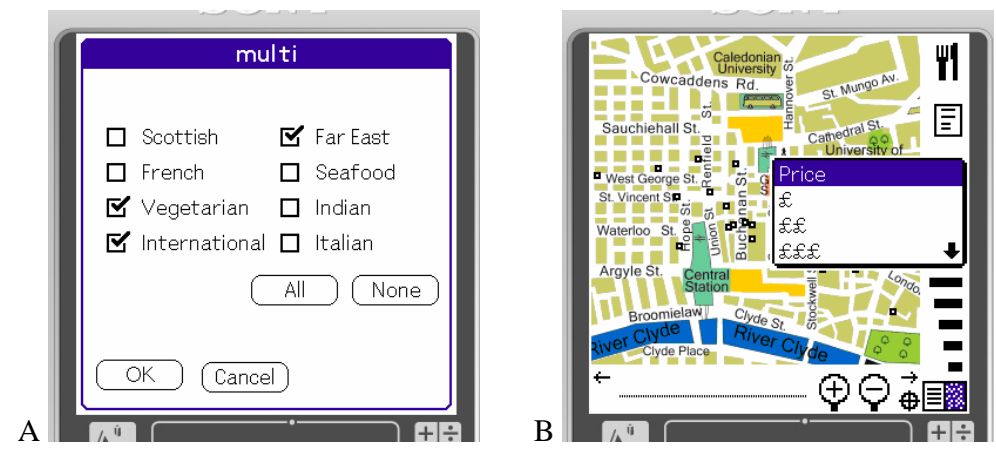

Fig. 3. Restaurant query filters (A: restaurant type, B: price band)

Starfield display technology has been proven to provide quick, dynamic and easy access to large amounts of data on desktop displays for both map-based information and more abstract data using simple X-Y scatterplots (Ahlberg.and Shneiderman, 1994). Starfield displays have also been shown suitable for accessing abstract data collections on palmtop computers (Dunlop and Davidson, 2000). The scatter plot does not only allow quick browsing through a desired subset of attractions but provides 
solutions to many problems of traditional searching: for example if helping users understand why they have no matching point displayed and showing locations of clusters within the data. Again Brown and Chalmers note that tourists often prefer to choose an area with more than one potential facility, for example heading into a "restaurant zone" of a city, often with one restaurant in mind but with flexibility to go elsewhere if that restaurant proves to be busy or unsuitable. Tourists can quickly look and browse through a cluster of facilities in particular areas before heading towards a specific establishment.

\section{Dynamic Content}

Tourist sites quite often change or can be temporarily closed. Museums and galleries have temporary exhibitions or host special events. Venues such as concert halls, theatres and cinemas change their program regularly on a weekly or monthly basis. It is desired to provide current and up to date information to tourists, so they can fully participate in the cultural life of a city, by not only seeing permanent attractions, buildings or monuments. Paper guidebooks cannot provide this kind of information due to their long publishing process. Taeneb City Guide uses a central database server to store its content including the data about all the attractions and associated events. It means that it is maintained centrally and can be easily administered and updated via a web-based interface. The data on mobile devices can be synchronised through a PC connected to the Internet via a Hot Synch process or using wireless Internet (Wi-Fi) at Hot Spots or via GSM or GPRS mobile phone networks. A future option may include providing synchronisation facilities at Tourist Information Centres or at popular tourist venues.

A very popular source of information used by tourist during their research for finding up-to-date date information before visiting a place is obviously the Internet. Taeneb City Guide provides a web-based interface (www.citygogo.com). The web interface was designed allowing the same set of functionalities as the PDA interface so it would help users to learn how to use the application on the PDA. PC web-based access was designed as a complementary element of the whole systems having in mind users arranging their trips in advance from home and then downloading the relevant information and synchronising it with their PDA devices. The use of a core central database for synchronisation means that users' data is kept up-to-date transparently between palmtop and web views. 


\section{Community review- forum for exchanging tourist information}

One recommendation is for new tourist information technologies to support sharing comments and reviews on different tourist attractions (Brown and Chalmers, 2003; Woodruff, A., et al., 2001). Taeneb City Guide incorporates a users' review system, which is available on handheld devices as well as through the Internet. The review system was created in a similar manner as forum systems for exchanging information already existing on the Internet and links reviews with the descriptions of attractions included in the electronic guide. It extends tourist experience through sharing opinions and advices with others who intend to visit a city. For tourist planning activities and collecting information this informal type of information is found very useful and according to conducted interviews was even better than information given by guidebooks or tourist staff (Crang 1999). Furthermore, the use of electronic guide allows reviews to be filtered so that, say, a user is initially only presented with reviews from peer-users using demographic information (e.g. young students would see reviews from other young students while middle-aged business travellers would see reviews for their peers).

An end-user can rate each attraction by selecting a rating from a pop-up scale menu and can add a text review (see Figure 4). Each review is by default private, to be stored for personal reference only, but can be marked as "Publish" making it public to be shared with other users. During the synchronisation process new reviews are uploaded and downloaded to mobile device.

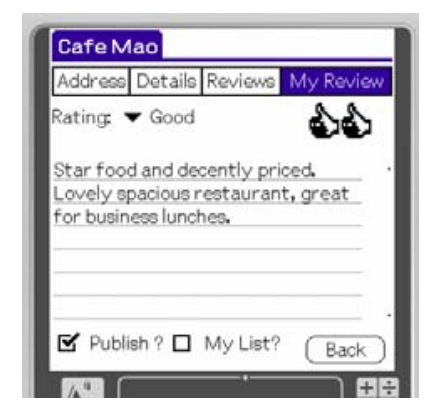

Fig. 4. Review attraction view 


\section{Usability trials}

Following the development of Taeneb City Guide System an initial usability trial was conducted with visitors attending an international conference in Glasgow. Twenty users were selected from the conference delegates, each of whom was lent a Sony Clié PEG-SL10 PalmOS devices (a device with a high-resolution but, unfortunately, only greyscale screen and rather poor contrast), and some other users who had their own PalmOS devices downloaded our software for the trial period. During the trial the available dataset was attractions covering restaurants, pubs, cinemas, theatres and accommodations for Glasgow city centre and included up-to-date "what's on" information. At the conference venue the participants were provided with access to synchronise the software and were encouraged to do so on at least a daily basis. The feedback was gathered after 2 day of trial in a form of informal interviews.

A lot of interest in the application was shown by people who had experience of palmtop computers / PDAs and by those interested in high-tech applications. People who had other than PalmOS devices, mostly PocketPC-based, expressed considerable disappointment that they could not download Taeneb City Guide to their own palmtop. One user found our PDA device in general too small and difficult to read and gave up using the system after the first day of trial. In general most of the users found the interface easy to use and intuitive even those who had never used a PDA before and did not want to spend too much time trying to figure out how to use another electronic gadget (all delegates were also participating heavily in the conference activities). They used the application mostly for searching for restaurants and they appreciated an easy way of finding a restaurant based on a location displayed on a map. They found the review system very helpful during their selection of restaurant and they were very pleased that they could use opinion of other delegates attending the conference but also stressed the importance of "professional" reviews being included along side community reviews. Most users added their own reviews of places they visited. Local users, i.e. those who lived in Glasgow, also found the system very useful - in particular the restaurants guide and cinema programs. One of the local users suggested adding an option allowing restaurants or cinema booking via GSM/SMS or GPRS network and others suggested that even information on restaurants that could normally cater for larger groups at short notice would be invaluable for conference delegates.

The overall feedback was positive with many suggestions of extending the data content (such as adding museums, galleries, train timetables etc.). Much of which could be achieved by augmenting the Taeneb interface either directly or through other palmtop software such as AvantGo channels. 


\section{Conclusions}

By analysing the requirements based on how people use traditional tourist publications we designed an electronic guide with an easy to use interface. All new distinctive elements of the system (dynamic query able map interface, synchronisation methods securing up to date content, and community review system) proved to give new advantages over traditional maps and guides. Although many people are still reluctant on using PDA type of devices in general, as the market of PDAs and smartphones grows (e.g. European PDA market saw 239 per cent increase in PDA shipments between 2002 and 2003 (Canalys, 2003)), there will be more and more users who would search for new software providing tourist information as a complementary source of information. Our user trials have given support for use of this complex tourist guide on small handheld computers.

\section{References}

Ahlberg, C. and Shneiderman, B. (1994). Visual Information Seeking: Tight Coupling of dynamic query filters with Starfield displays. Proceedings of CHI '94, 313-317 \& 479-480,

Brewster, S. A. and Dunlop, M. D. (2000). Human computer interaction with mobile devices. Editorial for special edition of Personal Technologies on Mobile HCI, vol. 4(2).

Brown, B. and M. Chalmers (2003). Tourism and Mobile Technology. Proc. Euro. Conf. Computer Supported Cooperative Work 2003 (ECSCW 2003)

Brown, B. and M. Perry (2002). Of maps and guidebooks: designing geographical technologies. Proceedings of Designing Interactive Systems (DIS) 2002. 246-254

Canalys (2003). Canalys research release 2003/071, http://www.canalys.com/pr/r2003071.htm [22 September 2003]

Dunlop, M. D. and Davidson, N. (2000). Visual information seeking on palmtop devices. Proceedings of HCI2000, volume 2, 19-20.

Woodruff, A., Aoki, P.M., Hurst, A., and Szymanski, M.H. (2001). Electronic Guidebooks and Visitor Attention. Proc. 6th Int'l Cultural Heritage Informatics Meeting, Milan, Italy, Sep., 437-454. 